\title{
The occurrence of Chlamydia spp. in pigs with and without clinical disease
}

Stina Englund ${ }^{1}$, Carl Hård af Segerstad ${ }^{1}$, Frida Arnlund ${ }^{2}$, Eva Westergren ${ }^{1}$ and Magdalena Jacobson ${ }^{3 *}$

\begin{abstract}
Background: Within the genera Chlamydia, the development of refined diagnostic techniques has allowed the identification of four species that are capable of infecting pigs. The epidemiology, clinical, and zoonotic impacts of these species are however largely unknown. The study aimed to investigate the presence of Chlamydia spp. in the intestines of growing pigs and in conjunctival swabs from finisher pigs, and relate the findings to clinical signs.

Results: By histology, 20 of 48 pigs had intestinal lesions that may be consistent with chlamydial infection. By PCR, forty-six of the pigs were positive whereas two samples were inhibited. Sequencing of 19 DNA extracts identified these as Chlamydia suis. By immunohistochemistry, 32 of 44 samples were positive and a significant relationship was detected between macroscopically visible intestinal lesions and a high degree of infection. By real-time PCR, a significant difference was detected between pigs with and without conjunctivitis when a Ct value of 36 was employed but not when a Ct value of 38 was employed.

Conclusions: Chlamydia suis was demonstrated in most samples and overall, no correlation to clinical signs was detected. However, a correlation was noted between samples with a high degree of infection and the presence of clinical signs. It is possible, that the intensive pig production systems studied might predispose for the transmission and maintenance of the infection thus increasing the infectious load and the risk for disease in the pig.
\end{abstract}

\section{Background}

The Chlamydiaceae family including the genera Chlamydia is a well-recognised cause of disease in many animal species including cats, ruminants, birds and humans $[1,2]$. Within the genera, nine distinct species have been identified. Four of these have been described in pigs (Chlamydia (C.) abortus, C. psittaci, C. pecorum, and C. suis) and related to reproductive disorders, conjunctivitis, enteritis, pneumonia, polyarthritis, pleuritis and polyserositis [1,3-5]. The epidemiology, clinical, and zoonotic importance of these species are however largely unknown [6-8]. However, C. suis seems to be both common and widespread, often occurring in mixed infections with C. abortus and C. pecorum [2].

In gnotobiotic pig challenge studies from USA, C. suis caused dose-dependent diarrhoea in young piglets. Histologically, villi atrophy, tip erosions, necrosis,

\footnotetext{
* Correspondence: Magdalena.Jacobson@kv.slu.se

${ }^{3}$ Department of Clinical Sciences, Faculty of Veterinary Medicine and Animal Science, Swedish University of Agricultural Sciences, P.O. Box 7054, 75007 Uppsala, Sweden

Full list of author information is available at the end of the article
}

inflammatory changes and lymphangitis were noted in the distal small intestine. Chlamydial antigens were demonstrated in enterocytes and chlamydiae were reisolated from tissue specimens and faecal swabs $[9,10]$. However, weaned pigs developed microscopical lesions but remained clinically healthy [11]. Similar lesions have also been demonstrated in clinical cases of diarrhoea in weaned pigs [12]. However, other studies have not been able to confirm a causal relationship $[3,8]$.

A few studies have addressed conjunctivitis [13]. Chlamydiae were seen in eight pigs by ultrastructural examination and were isolated in two pigs [4], and subclinical conjunctivitis was experimentally induced in 3-day-old gnotobiotic piglets [14].

Isolation is the gold standard for diagnosis but many strains are difficult to cultivate $[2,5]$. Other methods includes serology, immunohistochemistry, histology, and PCR on faeces, mucosal swabs or tissue specimens [2]. Because of cross-reactivity, some tests may lack sensitivity and specificity $[5,12,15]$. In a study comparing four diagnostic methods, C. suis were demonstrated in $42 \%$ of the samples by PCR and in $33 \%$ by culture. The 
sensitivity was $94.4 \%$ and specificity $81.0 \%$, whereas the two ELISAs performed considerably weaker. PCR was concluded to be a reliable, highly sensitive and specific tool for detection of C. suis [16].

The present study aimed to investigate the presence of Chlamydia spp in the intestines of growing pigs and in conjunctival swabs from finisher pigs, and relate those findings to the occurrence of clinical signs.

\section{Methods}

The study was approved by the Ethical Committee for Animal Experiments, Uppsala, Sweden. All herd owners had given an informed consent prior to the study.

\section{Herds and animals}

DNA from enteric specimens originated from a previous study on growing pigs with diarrhoea [17]. The samples originated from the major pig producing areas of Sweden, i.e. in the south-western and eastern parts of the country, and included 36 pigs from six herds with poor performance and diarrhoea in growing pigs, and 12 pigs from four herds with good performance and no diarrhoea. From each of the poor performance herds, three case and three control pigs were chosen, and from each of the four good performance herds, three control pigs were chosen. The case pigs had diarrhoea that had commenced within 2 days and no other diseases were evident. The control pigs were matched to the case pigs for age and sex, but showed no signs of clinical disease. The control pigs had a mean age of 72 days and a mean weight of $18.7 \mathrm{~kg}$, and the case pigs had a mean weight of $12.1 \mathrm{~kg}$. The pigs submitted from the good performance herds had a mean age of 67 days and a mean weight of $23.1 \mathrm{~kg}$. All animals were weaned at approximately 5 weeks of age and none of the pigs had been treated with antibiotics. The pigs were transported to the laboratory and euthanized within 15 min prior to necropsy.

Further, animals from three finisher herds were sampled based on the present occurrence of conjunctivitis. The pigs originated from several piglet-producing herds and were introduced to the finisher herds at 25$30 \mathrm{~kg}$. b.w., i.e. 4-14 weeks prior to sampling. They were kept 10 pigs per pen in units of 300 pigs each. In two herds, 7 case and 7 control pigs were selected from each of 3 units, and in one herd, 20 case and 20 control pigs from one unit were selected. The case pigs were selected based on clinical signs of moderate to severe conjunctivitis, defined as hyperaemia and chemosis with epiphora and/or muco-purulent secretion. The control pigs were selected from the same pens as the case pigs but showed no or only mild conjunctivitis, i.e. none to slight hyperaemia in the conjunctiva and no chemosis or epiphora.

\section{Necropsy and sampling}

The necropsies were carried out at the Department of Pathology at the National Veterinary Institute, Uppsala, or at AnalyCen, Skara. The animals were stunned with electricity, weighed and exsanguinated, and necropsy was immediately performed. All gross lesions were noted, and specimens for histological examination were taken from ileum and from all macroscopically visible lesions. The samples were fixed in $10 \%$ buffered formalin, embedded in paraffin blocks, sectioned and stained with haematoxylin and eosin according to standard protocols.

The finisher pigs were snared and sterile cotton swabs were rubbed against the conjunctiva in the conjunctival sac in the left and right eye, respectively. The swabs were placed in sterile tubes and transported to the laboratory.

\section{Polymerase chain reaction}

Intestinal samples were prepared from a $0.5 \mathrm{~cm}^{3}$ piece of frozen mucosa from the distal ileum. DNA was extracted by phenol/chloroform and precipitated by ethanol $[18,19]$. DNA from the conjunctival swabs was extracted according to K. Sachse [20] and all samples were analysed by real-time PCR according to Everett and others [21]. The primers TQF and TQR targeted the $23 \mathrm{~S}$ ribosomal DNA and detected all members of the family Chlamydiaceae. Multiple negative controls were included and DNA from Cp. abortus and C. suis (kindly provided by K. Sachse, Friedrich-Loeffler-Institute, Jena, Germany) were used as positive controls.

To detect false negative PCR results due to inhibiting agents, an internal amplification control (mimic) was constructed and used as previously described [22]. The primers used in the mimic producing PCR were TQFActin (5'GAAAAGAACCCTTGTTAAGGGAGCCATGTACCCTGGCATTG-3') and TQRActin (5'CTTAACTCCCTGGCTCATCATGGATCCACACGGAGTACTTGC-3'). The sequence of the ROX-labelled mimic probe used in real-time PCR was 5'-CCGACAGGATGCAGAAGGAGATCA-3'.

The conjunctival swabs were analysed both undiluted and diluted 1:100 in accordance with the standard protocol applied at the NVI, and a positive reaction in at least one of these dilutions was regarded as positive. In the statistical analyses, threshold values below Ct 36 were regarded as positive whereas values between $\mathrm{Ct} 36$ and $\mathrm{Ct} 38$ were regarded as doubtful. The results were calculated separately for the respective thresholds. Values above Ct 38 were regarded as negative or were excluded in the calculations. The difference in detection rate of Chlamydia spp. between pigs with moderate to severe conjunctivitis and the control pigs were analysed by chi-square test. 


\section{DNA sequence analysis}

Sequence analysis was performed on 19 samples from the intestinal specimens giving a strong positive reaction in the PCR. PCR was performed with primers 16SF2 and 23R according to Everett and Andersen [23] on DNA samples from seven case pigs and six control pigs from poor performance herds and six pigs from good performance herds. The predicted PCR product of 600 bp as well as one PCR product of larger and one of smaller size was purified prior to sequencing by using the GFX PCR DNA and Gel Band Purification Kit (Amersham Bioscience Europe, GmbH Germany). The purified products were sequenced with the same primers used in the PCR and by using the BigDye Terminator v3.1 Cycle Sequencing Kit (Applied Biosystems, Foster City, CA, USA) in combination with ethanol/EDTA/ sodium acetate precipitation, according to the protocol supplied. Thermocycling was performed in a GeneAmp 2700 Thermocycler (Applied Biosystems). The sequencing products were subjected to electrophoretic separation and on-line detection on an ABI PRISM 3100 Genetic Analyzer (Applied Biosystems), followed by BLAST search.

\section{Immunohistochemistry}

Paraffin-embedded intestinal specimens were available from 44 of the 48 growing pigs. $4-\mu \mathrm{m}$ thick sections of the formalin-fixed, paraffin-embedded blocks where cut and placed onto positively charged slides (Polysine ${ }^{\mathrm{TM}}$, Menzel-Gläser, Braunschweig, Germany). Prior to immunostaining, deparaffinisation and hydration where done in xylene and graded ethanol to distilled water. After hydration, a blocking for endogenous peroxidase where done in $0.03 \% \mathrm{H}_{2} \mathrm{O}_{2}$ in Tris-buffered saline (TBS) and nonspecific binding sites where blocked with $2 \%$ bovine serum albumin (BSA). Antigen retrieval was performed by heat induced epitope retrieval (HIER) in retrieval buffer $\mathrm{pH}=9$ (TBS-EDTA), using microwave as heat source. HIER was performed by heating the Polysine ${ }^{\mathrm{TM}_{-}}$-slides immersed in retrieval buffer for $7 \mathrm{~min}$ at $750 \mathrm{~W}$ followed by $14 \mathrm{~min}$ at $340 \mathrm{~W}$. After completed heating procedure, the slides remained in the retrieval buffer at room temperature for $20 \mathrm{~min}$. The slides where incubated with a mouse monoclonal antibody against Chlamydia, clone ACI (Progen Biotechnik Gmbh, Germany) diluted 1:100. Visualisation of the bound primary antibody was achieved by using Dako EnVision ${ }^{\mathrm{TM}}+$ System utilising an HCP-labelled antimouse monoclonal antibody (Glostrup, Denmark), and the presence of the relevant antigen was detected with 3,3'-diaminobenzidine (DAB, NVI, Sweden). Slides were weakly counterstained with haematoxylin. The staining included at least one positive and one negative control section. The positive control originated from previously confirmed routine cases. The results were graded as negative $(-)$, sparse $(+)$, moderate $(++)$ or abundant $(++$ + ) occurrence.

\section{Results}

\section{Necropsy}

No gross lesions were observed in the pigs from the good performance herds. In the pigs from the poor performance herds, five control pigs and 20 pigs with diarrhoea had macroscopic lesions consistent with Lawsonia (L.) intracellularis infection and one had a parasitic colitis. By histology, lesions that may be consistent with chlamydial infection [9] were noted in 20 pigs: Villus atrophy was noted in six pigs with diarrhoea and in ten control pigs from the poor performance herds. Epithelial exocytosis or necroses were noted in five pigs with diarrhoea, in one control pig from the poor performance herds, and in one pig from the good performance herds (Table 1).

\section{Microbiological analyses}

In the previous study [17], Brachyspira pilosicoli were demonstrated in eight case pigs and five control pigs, Campylobacter jejuni; in one case pig and four control pigs, Yersinia enterocolitica in three case and five control pigs, haemolytic Escherichia (E.) coli in two case and two control pigs, Clostridium perfringens in two case pigs, and L. intracellularis were previously demonstrated in 11 case and eight control pigs from the poor performance herds. In addition, haemolytic E. coli had been demonstrated in two pigs from the good performance herds. Rotavirus had been demonstrated in one case pig. Coronavirus was not included in the study, since Sweden has previously been shown to be free from

Table 1 The findings in intestinal specimens from growing pigs with diarrhoea (case), clinically healthy control pigs from the same poor performance herds (casecontrol), and from healthy pigs originating from good performance herds (control), examined by immunohistochemistry (IHC), necropsy, and PCR.

\begin{tabular}{lllll}
\hline & & $\begin{array}{l}\text { Case } \\
(\mathbf{n}=\mathbf{1 8})\end{array}$ & $\begin{array}{l}\text { Casecontrol } \\
(\mathbf{n}=\mathbf{1 8})\end{array}$ & $\begin{array}{l}\text { Control } \\
\mathbf{( n = 1 2 )}\end{array}$ \\
\hline IHC & - & $1(7 \%)$ & $9(53 \%)$ & $2(17 \%)$ \\
& + & $5(33 \%)$ & $3(18 \%)$ & $7(58 \%)$ \\
& ++ & $7(47 \%)$ & $4(24 \%)$ & $3(25 \%)$ \\
& +++ & $2(13 \%)$ & $1(6 \%)$ & 0 \\
\multirow{4}{*}{ Necropsy } & missing & 3 & 1 & - \\
\multirow{4}{*}{ PCR } & Villi atrophy & $6(33 \%)$ & $10(56 \%)$ & 0 \\
& Villi necrosis & $5(28 \%)$ & $1(6 \%)$ & $1(8 \%)$ \\
& positive & $18(100 \%)$ & $18(100 \%)$ & $10(83 \%)$ \\
& inhibited & 0 & 0 & $2(17 \%)$ \\
\hline
\end{tabular}

The percentage given within brackets is calculated on the actual number of analyses performed in each group, i.e. the "missing samples" are excluded 
transmissible gastroenteritis and porcine epidemic diarrhoea. Balantidium coli were demonstrated in one case and three control pigs from the poor performance herds, and in one pig from the good performance herds.

\section{Real-time PCR and DNA sequence analysis}

Of the 48 enteric samples analysed, 46 were positive for Chlamydiaceae (Table 1). Two samples could not be evaluated due to amplification inhibition. The major band of 600 b.p. found in all samples was confirmed as C. suis in the 19 samples subjected to sequence analysis. The larger PCR product described in the Methods' section was $98-100 \%$ identical to Escherichia coli whereas the smaller PCR product did not match any sequence in the BLAST search.

In the PCR analyses on the conjunctival swabs, one pig with conjunctivitis and three control animals together with their matched counterparts were excluded from the statistical calculations, since the PCR was partially or totally inhibited. Thus, in total, 116 conjunctival swabs were included in the statistical analyses. Of these, three samples from pigs with conjunctivitis gave weakly positive reactions ( $\mathrm{Ct}>38$ in undiluted samples) and were judged as negative.

Employing a cut-off value of $\mathrm{Ct} 36,45$ samples from pigs with conjunctivitis and 35 control pigs were determined as positive by PCR. Using a cut-off value of $\mathrm{Ct}$ 38, 48 samples from pigs with conjunctivitis and 42 control pigs were determined as positive. A statistically significant difference $(P=0.03)$ was detected between pigs with and without conjunctivitis when a $\mathrm{Ct}$ value of 36 was employed. The difference was mainly related to one of the herds (18 positive pigs with conjunctivitis and 12 positive control pigs). When a Ct of 38 was employed, no significant differences were noted.

\section{Immunohistochemistry}

The results are shown in Table 1. Among the case pigs, $6(40 \%)$ were negative or were carrying a low-grade infection. Among the control pigs from good performance herds, 9 (75\%) were negative or sparsely $(+)$ infected. The two control specimens in which the PCR analyses had been inhibited were graded as + and ++ , respectively, by immunohistochemistry. A significant ( $P$ $<0.05)$ relationship was detected between the presence of clinical signs and a high degree $(++$ or +++$)$ of infection. No relationship was detected between the occurrence of histological lesions and the demonstration of chlamydial antigen by immunohistochemistry.

\section{Discussion}

The pig intestine is considered as the natural reservoir for C. suis, and the microbe seems generally to be well adapted to its host [1]. In the present study, Chlamydia spp. were demonstrated in high prevalence in all herds investigated. This is consistent with the few studies that have previously addressed the occurrence of Chlamydia spp. in pig herds. Overall, it was not possible to relate the demonstration of the microbe to the presence of diarrhoea. However, based on the immunohistochemistry, pigs with diarrhoea might have been more heavily infected than the healthy pigs. This is in consistency with the results from another study on 447 pigs submitted for necropsy, where it was not possible to relate the presence of Chlamydia spp. to the occurrence of diarrhoea, but in 12 cases, it was the only pathogen found [6]. Similar experiences have also been noted in calves and sheep [24-26].

In the study by Becker et al. [13], C. suis was significantly $(P<0.0001)$ related to conjunctivitis in extensive pig production systems, whereas in intensive farming systems, high prevalences (88-90\%) were found in both pigs with conjunctivitis and in clinically asymptomatic pigs. This is in accordance with the findings in the present study. Becker et al. discussed, that environmental factors might predispose to infection. However, in one of the herds in the present study, factors such as emission of ammonium and carbon dioxide gases, air movements and overcrowding were investigated, but it was not possible to relate any environmental factor to the occurrence of conjunctivitis (data not shown). However, in one stable a high relative humidity $(83 \%)$ was noted that might facilitate microbial survival. In the present study, the pigs originated from several piglet producing herds and it should be emphasized that intensive farming systems with the mixing of pigs from several sources also imply increased opportunities for microbial spread among animals of different immunological status. In fact, in one herd a significant relationship was noted between conjunctivitis and the presence of Chlamydia spp. when the lower threshold value was applied in the real-time PCR. This might further indicate that the infectious load is important in the development of disease.

Although Chlamydia spp. was demonstrated in the ileal sections by immunohistochemistry in the present study, lesions compatible to those described in gnotobiotic pigs [9] were only noted in $50 \%$ of the pigs. Several authors speculate that the development of lesions may depend on different factors such as the virulence of the strain, the infectious dose, the route of infection, or the age and the immunological status of the host $[1,6,8,10,11,27]$. Since young, naive pigs seem to develop lesions in response to an experimental infection [9], host immunity might be induced at an early age in the field $[11,28]$. It is also possible that co-infections with other presumptive pathogens might act synergistically to exacerbate the lesions or that lesions induced by one 
microbe might increase the susceptibility to other infections. Several pathogens have been discussed in this respect $[3,6,11,13,29,30]$. Most of the intestinal lesions described in the present study were shown to be related to infections with L. intracellularis or B. pilosicoli [17].

Some studies also report the occurrence of mixed infection with several chlamydial species [3,31-33]. In wild boars, $C$. psittaci was the dominating species but $C$. suis and C. abortus was also demonstrated by sequencing [31]. In the present study, preliminary data obtained by PCR-RFLP indicated the co-infection by other species [34]. However, sequencing of the amplicons revealed the involvement of $C$. suis only, whereas other bands detected by PCR originated from E. coli and un-identified bacterial species. The results underline the difficulties involved in the diagnosis and a large variation in reported prevalences between various detection methods exist $[3,15,16,35]$. In the present study, the problem was circumvented by the use of an internal probe and a high melting temperature in the real-time PCR, combined with sequencing of the amplicons that assured a high specificity.

\section{Conclusions}

Chlamydia suis was found in high prevalences in growing pigs with or without diarrhoea, and in finisher pigs with or without conjunctivitis. Overall, no correlation to clinical signs was detected. However, a correlation was noted between samples with a high degree of infection and the presence of clinical signs. It is possible, that the intensive pig producing systems investigated in the present study might predispose for the transmission and survival of the microbe, thus increasing the infectious load and the risk for disease in the pig. Interestingly, no other species of the Chlamydiaceae family were detected, as also supported by the findings in other animal species in Swedish surveys [36].

\section{Acknowledgements}

We wish to thank Marianne Persson for skilful technical assistance. We also wish to thank the herd owners who kindly supplied the pigs and allowed us to enter the herds.

\section{Author details}

${ }^{1}$ National Veterinary Institute, 75189 Uppsala, Sweden. ${ }^{2}$ Stavby-Väsby 86, 747 94 Alunda, Sweden. ${ }^{3}$ Department of Clinical Sciences, Faculty of Veterinary Medicine and Animal Science, Swedish University of Agricultural Sciences, P. O. Box 7054, 75007 Uppsala, Sweden.

\section{Authors' contributions}

SE was responsible for the PCR investigation and sequencing of DNA from the intestinal specimens, analysis and interpretation of these data, and revised the manuscript. CHS made an intellectual contribution by drafting the manuscript and revising it critically. FA was responsible for collecting of the conjunctival swabs, PCR analysis and interpretation of these data, and revised the manuscript. EW was responsible for the immunohistochemical analyses, interpretations of these data, and revised the manuscript. MJ was planning the studies, collected and prepared the intestinal specimens, and was responsible for drafting and writing the manuscript. All authors read and approved the final manuscript.

\section{Competing interests}

The authors declare that they have no competing interests.

Received: 16 September 2011 Accepted: 26 January 2012

Published: 26 January 2012

\section{References}

1. Shewen PE: Chlamydial infection in animals: a review. Can Vet J 1980, 21:2-11.

2. Schautteet K, Vanrompay D: Chlamydiaceae infections in pig. Vet Res 2011, 42:1-10.

3. Hoelzle LE, Steinhausen G, Wittenbrink MM: PCR-based detection of chlamydial infection in swine and subsequent PCR-coupled genotyping of chlamydial omp--gene amplicons by DNA-hybridization, RFLPanalysis, and nucleotide sequence analysis. Epidemiol Inf 2000, 125:427-439.

4. Rogers DG, Andersen AA, Hogg A, Nielsen DL, Huebert MA: Conjunctivitis and keratoconjunctivitis associated with chlamydiae in swine. J Am Vet Med A 1993, 203(9):1321-1323.

5. Longbottom D: Chlamydial infections of domestic ruminants and swine: new nomenclature and new knowledge. Vet J 2004, 168:9-11.

6. Nietfeld JC, Leslie-Steen P, Zeman DH, Nelson D: Prevalence of intestinal chlamydial infection in pigs in the midwest, as determined by immunoperoxidase staining. Am J Vet Res 1997, 58(3):260-264.

7. Szeredi L, Schiller I, Sydler T, Guscetti F, Heinen E, Corboz L, Eggenberger E, Jones GE, Pospischil A: Intestinal Chlamydia in finishing pigs. Vet Pathol 1996, 33(4):369-374.

8. Zahn I, Szeredi L, Schiller I, Straumann Kunz U, Bürgi E, Guscetti F, Heinen E, Corboz L, Sydler T, Pospischil A: Immunohistologischer Nachweis von Chlamydia psittaci/pecorum und C. trachomatis im Ferkel-Darm. J Vet Med B 1995, 42:266-276.

9. Rogers $D G$, Andersen $A A$ : Intestinal lesions caused by two swine chlamydial isolates in gnotobiotic pigs. J Vet Diagn Invest 1996, 8:433-440.

10. Guscetti F, Schiller I, Sydler T, Heinen E, Pospischil A: Experimental enteric infection of gnotobiotic piglets with Chlamydia suis strain S45. Vet Microbiol 2009, 135:157-168.

11. Rogers DG, Andersen AA: Intestinal lesions caused by a strain of Chlamydia suis in weanling pigs infected at 21 days of age. J Vet Diagn Invest 2000, 12:233-239.

12. Nietfeld JC, Janke BH, Leslie-Steen P, Robison DJ, Zeman DH: Small intestinal Chlamydia infection in piglets. J Vet Diagn Invest 1993, 5:114-117

13. Becker A, Lutz-Wohlgroth L, Brugnera E, Lu ZH, Zimmermann DR, Grimm F, Grosse Beilage E, Kaps S, Spiess B, Pospischil A, Vaughan L: Intensively kept pigs pre-disposed to chlamydial associated conjunctivitis. J Vet Med A 2007, 54:307-313.

14. Rogers DG, Andersen AA: Conjunctivitis caused by a swine Chlamydia trachomatis-like organism in gnotobiotic pigs. J Vet Diagn Invest 1999, 11:341-344.

15. Rohde G, Straube E, Essig A, Reinhold P, Sachse K: Chlamydial zoonoses. Dtsch Ärtzebl Int 2010, 107(10):174-180.

16. Sachse K, Grossmann E, Jäger C, Diller R, Hotzel H: Detection of Chlamydia suis from clinical specimens: comparison of PCR, antigen ELISA, and culture. J Microbiol Meth 2003, 54:233-238.

17. Jacobson M, Hård af Segerstad C, Gunnarsson A, Fellström C, de Verdier Klingenberg $K$, Wallgren $P$, Jensen-Waern M: Diarrhoea in the growing pig-a comparison of clinical, morphological and microbial findings between animals from good and poor performance herds. Res Vet Sci 2003, 74:163-169.

18. Jacobson M, Aspan A, Heldtander Königsson M, Hård af Segerstad C, Wallgren P, Fellström C, Jensen-Waern M, Gunnarsson A: Routine diagnostics of Lawsonia intracellularis performed by PCR, serological and post mortem examination, with special emphasis on sample preparation methods for PCR. Vet Microbiol 2004, 102(3-4):189-201.

19. Johansson K-E, Persson A, Persson M: Diagnosis of contagious caprine and contagious bovine pleuropneumonia by PCR and restriction enzyme analysis. Int Symp Diagn Contr Livestock Dis Nucl Tech Int Atomic Energy Agency: 1998 Vienna, Italy; 1998, 137-158. 
20. Sachse K, Hotzel H: Detection and differentiation of Chlamydiae by nested PCR. Meth Mol Biol 2003, 216:123-136.

21. Everett KD, Hornung $L$, Andersen AA: Rapid detection of the Chlamydiaceae and other families in the order Chlamydiales: three PCR tests. J Clin Microbiol 1999, 37(3):575-580.

22. Englund S, Bölske G, Ballagi-Pordány A, Johansson K-E: Detection of Mycobacterium avium subsp. paratuberculosis in tissue samples by single, fluorescent and nested PCR based on the IS90 gene. Vet Microbiol 2001 81:257-271.

23. Everett $K D$, Andersen $A A$ : Identification of nine species of the Chlamydiaceae using PCR-RFLP. Int J Syst Bacteriol 1999, 49:803-813.

24. Storz J, Eugster AK, Altera KP, Olander HJ: Behavior of different bovine chlamydial agents in newborn calves. J Comp Pathol 1971, 81:299-307.

25. Doughri AM, Young S, Storz J: Pathologic changes in intestinal chlamydial infection of newborn calves. Am J Vet Res 1974, 35(7):939-944.

26. Lenzko H, Moog U, Henning K, Lederbach R, Diller R, Menge C, Sachse K, Sprague LD: High frequency of chlamydial co-infections in clinically healthy sheep flocks. BMC Vet Res 2011, 7:29[http://www.biomedcentral. com/1746-6148/7/29].

27. Reinhold P, Kirschvink N, Theegarten D, Berndt A: An experimentally induced Chlamydia suis infection in pigs results in severe lung function disorders and pulmonary inflammation. Vet Res 2008, 39:1-19.

28. Guscetti F, Schiller I, Sydler T, Corboz L, Pospischil A: Experimental Chlamydia psittaci serotype 1 enteric infection in gnotobiotic piglets: Histopathological, immunohistochemical and microbiological findings. Vet Microbiol 1998, 62:251-263.

29. Pospischil A, Wood RL: Intestinal Chlamydia in pigs. Vet Pathol 1987 24:568-570.

30. Koudela B, Vitovec J, Sterba J: Concurrent infection of enterocytes with Eimeria scabra and other enteropathogens in swine. Vet Parasitol 1990 35:71-77.

31. Hotzel H, Berndt A, Melzer F, Sachse K: Occurrence of Chlamydiaceae spp. in a wild boar (Sus scrofa L.) population in Thuringia (Germany). Vet Microbiol 2004, 103:121-126.

32. Schiller I, Koesters R, Weilenmann R, Thoma R, Kaltenboeck B, Heitz P, Pospischil A: Mixed infections with porcine Chlamydia trachomatis/ pecorum and infections with ruminant Chlamydia psittaci serovar 1 associated with abortions in swine. Vet Microbiol 1997, 58:251-260.

33. Pantchev A, Sting R, Bauerfeind R, Tyczka J, Sachse K: Detection of all Chlamydophila and Chlamydia spp. of veterinary interest using speciesspecific real-time PCR assays. Comp Immunol, Microbiol Inf Dis 2010, 33(6):473-484.

34. Jacobson M, Englund S: The occurrence of Chlamydia species in intestina specimens from Swedish grower pigs. The 20th International Pig Veterinary Society Congress: 2008 Durban, South Africa; 2008, 186.

35. Wendt M, Eggermann G, Wittenbrink MM, Jäger C, Weiss R, Failing K: Prevalence of Chlamydial infection in breeding sows. Proceedings of the 15th International Pig Veterinary Society Congress: 1998 Birmingham, England; 1998, 379

36. Godin A-C, Björkman C, Englund S, Johansson K-E, Niskanen R, Alenius S: Investigation of Chlamydophila spp. in dairy cows with reproductive disorders. Acta Vet Scand 2008, 50:39[http://www.actavetscand.com/ content/50/1/39].

doi:10.1186/1746-6148-8-9

Cite this article as: Englund et al:: The occurrence of Chlamydia spp. in pigs with and without clinical disease. BMC Veterinary Research 2012 8:9.

\section{Submit your next manuscript to BioMed Central and take full advantage of:}

- Convenient online submission

- Thorough peer review

- No space constraints or color figure charges

- Immediate publication on acceptance

- Inclusion in PubMed, CAS, Scopus and Google Scholar

- Research which is freely available for redistribution

Submit your manuscript at www.biomedcentral.com/submit 